\title{
The Importance of Leisure Activities in the Relationship between Physical Health and Well- Being in a Life Span Sample
}

\author{
Michelle E. Paggi ${ }^{a}$ Daniela Jopp ${ }^{c, d}$ Christopher Hertzog ${ }^{b}$ \\ ${ }^{a}$ New York City Department for the Aging, Research Unit, New York, N.Y., and b Georgia Institute of Technology, \\ School of Psychology, Atlanta, Ga., USA; ' University of Lausanne, Institute of Psychology, and ${ }^{\mathrm{d}}$ Swiss National \\ Centre of Competence in Research LIVES, Overcoming Vulnerability: Life Course Perspectives, \\ Lausanne, Switzerland
}

\section{Key Words}

Leisure $\cdot$ Physical health $\cdot$ Well-being $\cdot$ Successful aging

\begin{abstract}
Background: Previous studies have examined the relationships between physical health and leisure activities and between leisure activities and well-being, but, to our knowledge, none has examined these relationships simultaneously. Objective: This study investigated the relationships between leisure activities, health and well-being considering the role of age, and whether leisure activities mediate the relationship between physical health and well-being. Methods: Utilizing a cross-sectional database of 259 adults (ages 18-81 years) who completed several questionnaires, linear regression models and mediation models were tested. $\boldsymbol{R} \boldsymbol{e}$ sults: Regression analyses indicated that physical health was related to leisure activities and leisure activities were related to well-being. When physical health was measured by subjective ratings, age had a stronger relationship with leisure activities. However, when physical health was indicated by health restrictions, physical health had a stronger relationship with leisure activities than did age. Leisure activities were a partial mediator of the relationship between physical health and well-being. Conclusion: The results demonstrat-
\end{abstract}

ed that the reduction in leisure activities with age has more to do with physical health limitations than with older age itself. In addition, regardless of age, the benefits of physical health for well-being are due in part to the level of leisure activity participation. These results highlight the importance of leisure activities for successful aging throughout the adult life span. Interventions designed to improve well-being through increasing leisure activity participation should take physical health into consideration, particularly for older adults.

(c) 2016 S. Karger AG, Basel

\section{Introduction}

Participation in leisure activities is associated with various components of successful aging, including physical health and well-being [1]. Leisure activities are broadly defined as activities, such as exercise and socializing, individuals take part in during free time [2]. It has been argued that leisure activities and psychosocial factors (e.g., well-being) are antecedents of successful aging [3]. However, aspects of successful aging may also precede participation in leisure activities and experiencing well-being: those who age successfully remain healthy enough to 
engage in leisure activities and in turn likely to experience more positive psychological outcomes. Thus, models of successful aging should account for constraints imposed by disease and disability [4]. Furthermore, there has been a recent push to consider the extent to which individuals have access to successful aging-promoting measures (e.g., quality health care) given social inequalities [5].

There is evidence that physical health affects participation in leisure activities and that both physical health and activities affect well-being [6-8]. Health is probably a necessary but not sufficient condition for participation in some kinds of leisure activities; there are other variables that may influence engagement in leisure activities (e.g., cognitive functioning or personality [9]). Nevertheless, the current study focuses on health and well-being as key aspects related to leisure activities. Specifically, we propose that leisure activities are a key link between physical health and well-being. The finding that leisure activities mediate the relationship between health and well-being could have important implications for health recommendations to improve psychological outcomes.

\section{Leisure Activities and Physical Health}

In the current study, we used self-report indicators of physical health (or subjective health), including overall health, functional status, and presence of chronic health problems. Previous studies have shown that subjective physical health predicts the extent to which individuals can engage in leisure activities. Both cross-sectional and longitudinal studies have found that age-related functional decline not only reduced the reported frequency of physical activity participation [7, 10-13] but also determined the types of physical activities one could participate in, such as sports and yard work [14]. Research has shown that neurological illnesses affect engagement in physical activities. Hildebrand et al. [15] found that older adults who suffered a mild stroke significantly reduced their number of moderate- to high-intensity physical activities 6 months after stroke. In another study, older adults were more likely to meet UK guidelines for moderate or vigorous physical activity if they had fewer chronic health problems and severe mobility impairments [16]. Physical health not only affects the frequency, but also the intensity of physical activities. Taylor-Piliae et al. [8] found that higher physical well-being was significantly related to older adults' intensity of physical activity. Taken together, these studies suggest that various areas of physical health may affect one's ability to engage in physical activities.

Leisure Activities, Physical Health, and Well-Being
Physical health also affects participation in nonphysical activities: Lawton et al. [6] found that older adults reporting a greater number of health conditions engaged in more other-directed projects (i.e., community-centered and/or altruistic in nature). Janke et al. [11] found in a sample of adults aged 50 and older that socializing with others significantly declined with increasing functional limitations and chronic illnesses. Taken together, these studies show that physical health can enable individuals to engage in or preclude them from taking part in various kinds of leisure activities. However, the effect of physical health on leisure activity participation has been studied mostly in older individuals and few studies have examined the relationship between physical health and leisure activities that are not physical in nature. The current study closes this knowledge gap by investigating the effects of physical health on a wide variety of leisure activities in young, middle-aged, and older adults.

\section{Leisure Activities and Well-Being}

One of the most common indicators of well-being in the psychological literature is life satisfaction; thus, this construct was chosen to measure well-being in the current study. Previous studies have demonstrated that leisure activities can improve well-being. Using a sample representing a wide age range (19-89 years), Pressman et al. [2] found that well-being was positively associated with the frequency of participating in enjoyable leisure activities (e.g., spending quiet time alone, socializing with others, and hobbies), highlighting the importance of various kinds of leisure activities throughout the adult life span. A relationship between leisure activities and wellbeing was also shown in a study of older adults in Taiwan in which a variety of activity types were measured [17].

Longitudinal studies have also demonstrated that various kinds of leisure activities positively impact well-being. Menec and Chipperfield [7] found that the level of participation in leisure activities predicted life satisfaction 7 years later in a sample of older adults; a similar longitudinal study replicated these findings [18]. Kahana et al. [19] found that older adults who engaged in more social activities, such as volunteering and informal helping behavior, had greater life satisfaction 3 years later. Van Willigen [20] demonstrated that older adults who volunteered more hours had greater life satisfaction 3 years after baseline. Thus, there is evidence from both cross-sectional and longitudinal studies for the value of a wide range of leisure activities for psychological well-being, indicating that this relationship is both cohort- and timeindependent. 
Physical Health and Well-Being

Finally, there is evidence from previous studies using culturally diverse samples to support the relationship between physical health and well-being across the adult life span. Survey studies of undergraduates representing various Western and Eastern cultures indicated that greater well-being was associated with various health-related behaviors (e.g., not smoking, exercise, and getting the recommended amount of sleep) and higher physical functioning [21-23]. This relationship has also been demonstrated in samples including middle-aged and older adults. A study of adults aged 18-66+ years living in rural Australia found that those with lower physical disability had higher odds of having life satisfaction [24]. In a survey study utilizing a sample of over 430,000 adults aged 18-65+ years across the United States, physical health emerged as one of the strongest predictors of life satisfaction, along with unemployment, disability, and social relationships; these results demonstrate that physical health is on a par with other major antecedents of well-being in the adult life span [25]. Taken together, these studies indicate that physical health is consistently related to wellbeing across a variety of age groups and cultures. The current study sought to determine whether leisure activities are a mechanism through which physical health impacts well-being.

\section{The Present Study}

The purpose of the current study was to investigate the relationships between physical health, leisure activities, and well-being in a life span sample. Previous studies support the hypothesized relationships between these three variables $[2,11,25]$, but, to our knowledge, none have tested these relationships simultaneously. The current study filled this knowledge gap investigating the concurrent links between leisure activity engagement, health limitations, subjective health, and well-being in a life span sample. Specifically, leisure activities were investigated as a mediator in the relationship between physical health and well-being.

Research evidence supporting the disengagement theory suggests that reduction in leisure activities is part of growing older [26]. However, because aging is characterized by heterogeneity, it is proposed that the relationship between age and leisure activity engagement differs by physical health status; in other words, healthier adults may experience a smaller decrease in leisure activities with age than less healthy adults. Though the positive association between age and well-being is well-documented, recent research indicates that individual older adults' well-being changes over time at different rates [27]. Thus, we examined whether the relationships between physical health, leisure activities, and well-being differed according to age. It was predicted that older individuals with higher levels of physical health and leisure activity engagement would have higher ratings of well-being than those with poorer physical health and low activity levels. Although we are aware that specific activities (e.g., social vs. physical activities) may have different relations to health and well-being, we decided to use general leisure activities as a whole, in line with classic approaches such as activity theory and a substantial body of literature on leisure activities, as reported above.

\section{Methods}

\section{Participants}

These cross-sectional data for the current study were originally collected for a study on the role of beliefs regarding cognitive aging. Five participants with incomplete data were excluded, resulting in a sample of 259 adults aged 18-81 years [mean age $=49.07$, $\mathrm{SD}=17.27$ ] [9]. Stratified sampling for age and gender resulted in approximately equal amounts of participants for each age decade and gender ( $46 \%$ male, $54 \%$ female). The sample consisted of white (61\%), African American (31\%) and Asian (5\%) participants, and $3 \%$ were of another ethnicity. Participants were recruited through television and newspaper advertisements, inviting them to participate in a study investigating beliefs about aging; they were paid for their participation [9].

\section{Measures}

Physical Health

Physical health was measured by two variables: subjective health and health restrictions. Subjective health was measured by having the participants rate their general health status on a Likerttype scale (from $1=$ excellent to 5 = poor). The health restriction variable was a composite score of participant ratings of activity limitations, number of diagnoses, and number of medications. This composite score was computed by calculating the mean of the $\mathrm{Z}$ scores of these variables. To measure activity limitations, participants were asked to rate how health issues affected the following activities: chores, getting around town, mental recreation, physical recreation, hobbies, and travel (the rating scale ranged from $1=$ strongly positive to $7=$ strongly negative [28]).

\section{Leisure Activities}

Leisure activities were assessed using an extended version of the Victoria Longitudinal Study activity questionnaire [9]; participants indicated their frequency of participation in 82 activities using the following 9-point Likert-type scale: $0=$ never, $1=$ less than once a year, 2 = about once a year, $3=2$ or 3 times a year, $4=$ about once a month, $5=2$ or 3 times a month, $6=$ about once a week, $7=2$ or 3 times a week, and $8=$ daily. The questionnaire was found to have a factor structure of 11 activity types: physical activities, games, crafts, watching TV, social-private, social-public, religious activities, developmental activities, experiential activities, technol- 
Table 1. Descriptive statistics for study variables

\begin{tabular}{|c|c|c|c|c|c|c|c|c|}
\hline & \multicolumn{2}{|c|}{$\begin{array}{l}\text { Total } \\
(\mathrm{n}=259)\end{array}$} & \multicolumn{2}{|c|}{$\begin{array}{l}\text { Young: } 18-30 \text { years } \\
(\mathrm{n}=46)\end{array}$} & \multicolumn{2}{|c|}{$\begin{array}{l}\text { Middle-aged: } 31-59 \text { years } \\
(\mathrm{n}=125)\end{array}$} & \multicolumn{2}{|c|}{$\begin{array}{l}\text { Older: } 60-81 \text { years } \\
(\mathrm{n}=88)\end{array}$} \\
\hline & mean & $\mathrm{SD}$ & mean & SD & mean & $\mathrm{SD}$ & mean & SD \\
\hline \multicolumn{9}{|l|}{ Sociodemographic } \\
\hline Age (years) & 49.1 & 17.3 & 23.8 & 3.6 & 44.7 & 8.5 & 68.4 & 5.6 \\
\hline Education (years) & 14.9 & 2.3 & 14.4 & 2.2 & 15.1 & 2.3 & 14.8 & 2.3 \\
\hline African American (\%) & 30.9 & & 30.4 & & 43.2 & & 13.6 & \\
\hline Asian (\%) & 4.6 & & 26.1 & & 0.0 & & 0.0 & \\
\hline Other (\%) & 3.1 & & 2.2 & & 3.2 & & 2.3 & \\
\hline \multicolumn{9}{|l|}{ Health } \\
\hline Subjective $^{\mathrm{a}}$ & 3.8 & 0.9 & 4.0 & 0.9 & 3.7 & 0.9 & 3.8 & 0.9 \\
\hline
\end{tabular}

${ }^{a}$ Higher scores indicate better health. ${ }^{\mathrm{b}}$ Higher scores indicate more restrictions due to health; values based on $\mathrm{Z}$ scores; all age groups significantly different from one another at $\mathrm{p}<0.01$ : younger $<$ middle-aged, younger $<$ older, middle-aged $<$ older. ${ }^{\mathrm{c}}$ Composite score representing the mean activity level across all domains; young and older age groups significantly different from each other at $\mathrm{p}<0.05$ : younger $>$ older. ${ }^{\mathrm{d}}$ Young and middle-aged and middle-aged and older groups significantly different from each other at $\mathrm{p}<0.01$ : younger $>$ middle-aged, middle-aged $<$ older.

ogy use, and travel. The score that was used in the analyses was a composite score of the means of all of the activity domains.

\section{Well-Being}

Well-being was measured using the Satisfaction with Life Scale [29]; participants rated their agreement with 5 statements (e.g., 'In most ways, my life is close to my ideal') on a Likert-type scale, ranging from 1 ('strongly disagree') to 5 ('strongly agree').

\section{Statistical Analyses}

SPSS version 20 was utilized for all analyses. Preparing the more complex regression analysis, we examined the zero-order relations among key study variables with Pearson product moment correlations (between continuous variables) and point biserial correlations (between binary and continuous variables). The hypotheses were then tested using linear regression analyses and $p$ values less than 0.05 were considered statistically significant. Separate regression models were run for both indicators of physical health. Age, gender, years of education, and race were included as covariates in the analyses because previous research has shown that they are related to physical health, activities, and well-being [25, 30, 31]. Race was dummy-coded to create two binary variables: African American ( 1 = African American, 0 = White or other $)$ and 'other' ( $1=$ other, $0=$ African American or White). The 'other' category included the subset of the participants $(4.6 \%)$ who did not identify themselves as either African American or White, all of whom were younger adults. Interactions between age and all of the independent variables were tested to determine whether there were agedifferential effects.

Leisure Activities, Physical Health, and Well-Being
Mediation was tested using the criteria by Preacher and Hayes [32]: (1) the path from the independent variable to the dependent variable must be statistically significant and (2) according to the Sobel test, the indirect effect of the independent variable on the dependent variable via the mediator must be statistically significant [32].

\section{Results}

\section{Descriptives and Correlations}

Table 1 shows means and standard deviations of the study variables for the total sample as well as separately for young, middle-aged, and older adults. Table 2 displays the correlations between the study variables. The two health indicators were significantly correlated $(r=-0.33$, $\mathrm{p}<0.01$ ), but not highly enough to suggest that these constructs were redundant. Therefore, it was worth investigating how both subjective health and health restrictions are individually related to leisure activities and well-being. As predicted, the correlations between the health indicators and leisure activities were significant (subjective health: $\mathrm{r}=0.25$; health restrictions: $\mathrm{r}=-0.31$; $\mathrm{p}$ values $<0.01)$ and were stronger than the correlation between age and leisure activities $(\mathrm{r}=-0.18, \mathrm{p}<0.01)$. As predict- 
Table 2. Intercorrelations of demographic, health, leisure activities, and well-being variables

\begin{tabular}{|c|c|c|c|c|c|c|c|c|}
\hline Variable & 1 & 2 & 3 & 4 & 5 & 6 & 7 & 8 \\
\hline \multicolumn{9}{|l|}{ (1) Age } \\
\hline (2) Education & 0.05 & & & & & & & \\
\hline (4) African American ${ }^{b}$ & $-0.22^{* *}$ & $-0.23^{* *}$ & 0.11 & & & & & \\
\hline (5) Other ${ }^{c}$ & $-0.28 * *$ & -0.02 & 0.00 & $-0.19 * *$ & & & & \\
\hline (8) Leisure activities & $-0.18^{* *}$ & $0.18^{* *}$ & -0.04 & $-0.19^{* *}$ & 0.08 & $0.25^{* *}$ & $-0.31^{* *}$ & \\
\hline (9) Well-being & $0.18^{* *}$ & $0.20^{* *}$ & -0.09 & $-0.28^{* *}$ & 0.11 & $0.35^{* *}$ & $-0.12^{*}$ & $0.24^{* *}$ \\
\hline
\end{tabular}

Table 3. Regression models with leisure activities as the criterion: separate models for different health indicators

\begin{tabular}{|c|c|c|c|c|}
\hline & \multicolumn{4}{|c|}{ Total $(n=259)$} \\
\hline & B & SE & $\beta$ & $\mathrm{R}^{2}$ \\
\hline Model 1a & & & & $0.14^{* *}$ \\
\hline Subjective health & 0.16 & 0.05 & $0.21^{* *}$ & \\
\hline Age & -0.04 & 0.00 & $-0.34^{* *}$ & \\
\hline Age $\times$ subjective health & -0.00 & 0.00 & -0.06 & \\
\hline Education & 0.02 & 0.02 & 0.08 & \\
\hline Age $\times$ education & 0.00 & 0.00 & 0.12 & \\
\hline Gender $^{\mathrm{a}}$ & 0.03 & 0.08 & 0.03 & \\
\hline Age $\times$ gender & 0.00 & 0.01 & 0.07 & \\
\hline African American ${ }^{b}$ & -0.29 & 0.10 & $-0.19^{* *}$ & \\
\hline Age $\times$ African American & 0.01 & 0.01 & $0.15^{*}$ & \\
\hline Other ${ }^{c}$ & -0.16 & 0.22 & -0.06 & \\
\hline Age $\times$ other & -0.00 & 0.01 & -0.01 & \\
\hline Model $1 b$ & & & & $0.15^{* *}$ \\
\hline Health restrictions ${ }^{\mathrm{d}}$ & -0.26 & 0.07 & $-0.27^{* *}$ & \\
\hline Age & -0.01 & 0.00 & $-0.22^{*}$ & \\
\hline Age $\times$ health restrictions & 0.01 & 0.00 & 0.10 & \\
\hline Education & 0.03 & 0.02 & 0.10 & \\
\hline Age $\times$ education & 0.00 & 0.00 & 0.10 & \\
\hline Gender & 0.04 & 0.08 & 0.03 & \\
\hline Age $\times$ gender & 0.00 & 0.01 & 0.05 & \\
\hline African American & -0.28 & 0.10 & $-0.19^{* *}$ & \\
\hline Age $\times$ African American & 0.01 & 0.01 & $0.15^{*}$ & \\
\hline Other & -0.18 & 0.22 & -0.07 & \\
\hline Age $\times$ other & 0.00 & 0.01 & 0.02 & \\
\hline
\end{tabular}

${ }^{\mathrm{a}} 0=$ male, 1 = female. ${ }^{\mathrm{b}} 0$ = Caucasian or other, $1=$ African American. ${ }^{\mathrm{c}} 0=$ African American or Caucasian $1=$ other ethnicity. ${ }^{\mathrm{d}}$ Higher values $=$ more restrictions due to health. ${ }^{*} \mathrm{p}<0.05$; ** $\mathrm{p}<0.01$. ed, leisure activities were also significantly correlated with well-being $(\mathrm{r}=0.24, \mathrm{p}<0.01)$, and this correlation was stronger than the correlation between age and wellbeing $(r=0.18, p<0.01)$. The sociodemographic variables (i.e., gender, education, and race) were also significantly correlated with certain health, activity, and well-being indicators, so it was crucial to include these variables in the regression models as covariates.

\section{Regression Analyses Investigating the Relationship \\ between Leisure Activities and Subjective Health}

The results of the regression analyses investigating the relationship between leisure activities and physical health indicators are shown in table 3. Model 1a, which included subjective health as an independent variable, explained $14 \%$ of the variance in leisure activities at $\mathrm{p}<0.01$. Age was the independent variable most strongly related to leisure activities $(\beta=-0.34, \mathrm{p}<0.01)$, indicating that participants engaged with fewer leisure activities with age. Subjective health was positively related to leisure activities $(\beta=0.21, p<0.01)$, and being African American was negatively related to leisure activities $(\beta=-0.19, \mathrm{p}<0.05)$. Age interacted in the positive direction with being African American $(\beta=0.15, p<0.01)$, indicating that, with age, African Americans engage in more leisure activities.

Model 1b, which included health restrictions as an independent variable, explained $15 \%$ of the variance in leisure activities at $\mathrm{p}<0.01$. Health restrictions had the strongest relationship with leisure activities $(\beta=-0.27$, $\mathrm{p}<0.01)$, followed by age $(\beta=-0.22, \mathrm{p}<0.01)$, and then by being African American $(\beta=-0.19, \mathrm{p}<0.01)$. As with model 1a, age interacted positively with being African American $(\beta=0.15, \mathrm{p}<0.01)$. 
Table 4. Regression models with well-being as the criterion: separate models for different health indicators

\begin{tabular}{|c|c|c|c|c|}
\hline & \multicolumn{4}{|c|}{ Total $(n=259)$} \\
\hline & B & SE & $\beta$ & $\mathrm{R} 2$ \\
\hline Model $2 a$ & & & & $0.23^{* *}$ \\
\hline Leisure activities & 0.27 & 0.13 & $0.13^{*}$ & \\
\hline Age & 0.02 & 0.01 & $0.22^{*}$ & \\
\hline Age $\times$ leisure activities & -0.00 & 0.01 & -0.03 & \\
\hline Subjective health & 0.45 & 0.09 & $0.28^{* *}$ & \\
\hline Age $\times$ subjective health & 0.01 & 0.01 & $-0.12^{*}$ & \\
\hline Education & 0.04 & 0.04 & 0.06 & \\
\hline Age $\times$ education & 0.01 & 0.00 & $0.13^{*}$ & \\
\hline Gender $^{\mathrm{a}}$ & -0.09 & 0.17 & -0.03 & \\
\hline Age $\times$ gender & -0.00 & 0.01 & -0.03 & \\
\hline African American ${ }^{b}$ & -0.40 & 0.20 & $-0.13^{*}$ & \\
\hline Age $\times$ African American & 0.02 & 0.01 & 0.08 & \\
\hline Other ${ }^{c}$ & 0.72 & 0.44 & 0.13 & \\
\hline Age $\times$ other & 0.01 & 0.02 & 0.03 & \\
\hline Model $2 b$ & & & & $0.19^{* *}$ \\
\hline Leisure activities & 0.33 & 0.13 & $0.16^{*}$ & \\
\hline Age & 0.02 & 0.01 & $0.28^{* *}$ & \\
\hline Age $\times$ leisure activities & -0.00 & 0.01 & -0.01 & \\
\hline Health restrictions ${ }^{\mathrm{d}}$ & -0.39 & 0.14 & $-0.19^{* *}$ & \\
\hline Age $\times$ health restrictions & 0.02 & 0.01 & 0.13 & \\
\hline Education & 0.07 & 0.04 & 0.11 & \\
\hline Age $\times$ education & 0.00 & 0.00 & 0.10 & \\
\hline Gender $^{\mathrm{a}}$ & -0.14 & 0.17 & -0.05 & \\
\hline Age $\times$ gender & -0.01 & 0.01 & -0.04 & \\
\hline African American ${ }^{\mathrm{b}}$ & -0.37 & 0.21 & -0.12 & \\
\hline Age $\times$ African American & 0.02 & 0.01 & 0.08 & \\
\hline Other & 0.75 & 0.45 & 0.14 & \\
\hline Age $\times$ other & 0.02 & 0.02 & 0.07 & \\
\hline
\end{tabular}

${ }^{\mathrm{a}} 0=$ male, $1=$ female. ${ }^{\mathrm{b}} 0=$ Caucasian or other, $1=$ African American. ${ }^{c} 0=$ African American or Caucasian, $1=$ other ethnicity. ${ }^{\mathrm{d}}$ Higher values $=$ more restrictions due to health. ${ }^{*} \mathrm{p}<0.05$, $* * \mathrm{p}<0.01$.

\section{Regression Analyses Investigating the Relationship} between Well-Being and Leisure Activities

The results of the regression analyses investigating the relationship between well-being, leisure activities, and physical health are displayed in table 4 . Model 2a, which included leisure activities and subjective health as independent variables, explained $23 \%$ of the variance in wellbeing at $\mathrm{p}<0.01$. Subjective health had the strongest relationship with well-being $(\beta=0.28, \mathrm{p}<0.01)$, followed by age $(\beta=0.22, \mathrm{p}<0.01)$, leisure activities $(\beta=0.13$, $\mathrm{p}<$ $0.05)$, and being African American $(\beta=-0.13, \mathrm{p}<0.05)$. Age interacted with subjective health in the negative di- rection $(\beta=-0.12, p<0.05)$, meaning at higher levels of subjective health, older age was related to lower well-being. Age also interacted with education in the positive direction $(\beta=0.13, \mathrm{p}<0.05)$, showing that at older ages, more education is related to higher well-being.

Model 2b, which included health restrictions and leisure activities as independent variables, explains $19 \%$ of the variance in well-being at $p<0.01$. Age had the strongest relationship with well-being $(\beta=0.28, \mathrm{p}<0.01)$, followed by health restrictions $(\beta=-0.19, \mathrm{p}<0.01)$, and leisure activities $(\beta=0.16, \mathrm{p}<0.05)$.

\section{Mediation Models}

Two mediation models were tested: leisure activities mediating the relationship between subjective health and well-being and leisure activities mediating the relationship between health restrictions and well-being. Both sets of regression analyses for the mediation models included age, education, gender, and race as covariates. Figure 1 shows the results of the first mediation model in which the criteria by Preacher and Hayes for mediation were met: subjective health was significantly related to wellbeing $(\beta=0.31, p<0.01)$ and, according to the Sobel test, the indirect effect of subjective health on well-being via leisure activities is significantly different from $0(Z=2.13$, $\mathrm{p}=0.03)$. However, because the relationship between subjective health and well-being was still significant when adding the mediator $(\beta=0.27, \mathrm{p}<0.01)$, leisure activities presented as a partial mediator.

For the second mediation model, the criteria by Preacher and Hayes for mediation were also met (fig. 2): health restrictions were significantly related to well-being $(\beta=-0.21, p<0.01)$ and, according to the Sobel test, the indirect effect of health restrictions on well-being via leisure activities is significantly different from $0(Z=-2.36$, $\mathrm{p}=0.02$ ). However, because the relationship between subjective health and well-being was still significant when adding the mediator $(\beta=-0.16, p<0.05)$, leisure activities also acted as a partial mediator. Thus, leisure activities play a significant role in the relationships between the perception of physical health and objective indicators of physical health and well-being.

\section{Discussion}

The purpose of the current study was to examine the links between physical health, leisure activities, and wellbeing as well as to determine how age affected the relationships between these variables. The first aim was to 


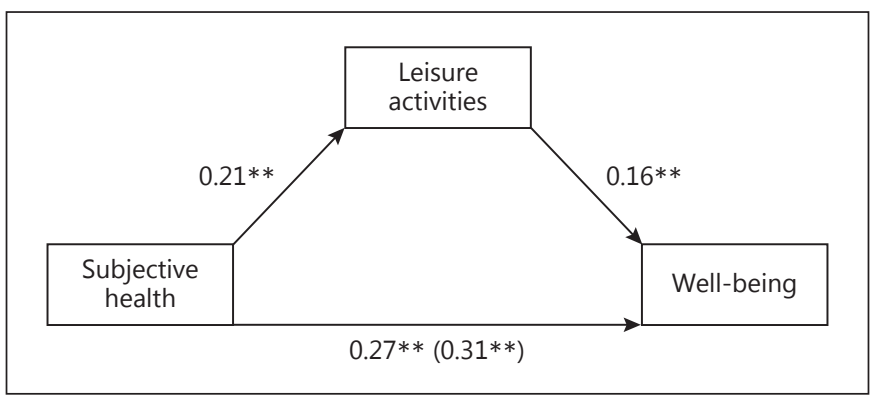

Fig. 1. Leisure activities partially mediate the relationship between subjective health and well-being. Results of the Sobel test: test statistic $=2.13$, standard error $=0.03, p=0.03$. Coefficient in parentheses: $\beta$ value of the predictor before the mediator was added to the model. ${ }^{* *} \mathrm{p}<0.01$.

determine the relationship between physical health and leisure activities and compare this relationship to the one between leisure activities and age. Both subjective health and health restrictions were related to the participation in leisure activities, replicating previous research findings in which different indicators of physical health were found to be related to leisure activities [2]. Being African American versus being white or of another race was associated with reporting fewer leisure activity engagements; however, with older age, African American participants engaged in more leisure activities. These findings replicate previous research showing that African Americans engage in less leisure time physical activity than white individuals, but this difference in activity participation decreases with age [30]. Shaw et al. [30] propose that the difference in activity levels between African American and white younger adults is due to disparities in the access to health-promoting activities; however, because white adults' activity levels decrease with age, these disparities level off over time and the African American older adults who live into old age are still alive due in part to their engagement in physical activities. Furthermore, in both the study by Shaw et al. and in our study, the activity assessments may not have adequately captured the types of activities younger African Americans participate in, so future studies should focus on developing leisure activity scales that are more inclusive of various cultures.

The current study also found that the function of age in the relationship between physical health and activities depended on how physical health was defined. When physical health is based on overall self-ratings, age played a greater role in leisure activity participation than physical health. When physical health was based on health re-

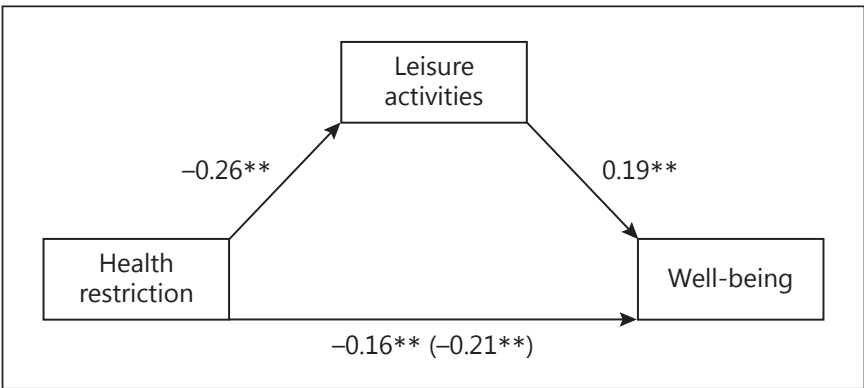

Fig. 2. Leisure activities partially mediate the relationship between objective health and well-being. Results of the Sobel test: test statistic $=-2.36$, standard error $=0.04, p=0.02$. Coefficient in parentheses: $\beta$ value of the predictor before the mediator was added to the model. ${ }^{* *} \mathrm{p}<0.01$.

strictions, representing a health indicator less biased by age-adjusted comparison standards [28], physical health played a greater role in leisure activity participation than age. These results suggest that it is rather the physical limitations that come with age than older age itself that explains why older individuals participate in fewer activities; this conclusion is further supported by the fact that age and health restrictions were correlated at $r=0.47$. In contrast, considering age and subjective health were not correlated $(r=-0.01)$, the effects of subjective health on leisure activity engagement operate independently from age. Previous research has found similar patterns, showing that even very old individuals such as centenarians report high subjective health, despite poor functional capacity and multimorbidity [33].

The second aim of the study was to determine the relationship between leisure activities and well-being and compare this relationship to the link between age and well-being. The results indicated that leisure activity participation was positively related to well-being, corroborating findings from previous research $[2,6,7,17,18,34]$. However, age had a stronger relationship with well-being than activities and this relationship was positive, indicating that participants experienced higher well-being with age. This finding makes sense in light of previous research demonstrating a positive relationship between age and well-being after controlling for functional health [35]. As expected, both physical health indicators were positively related to well-being, replicating previous research on the relationship between health and well-being [21, 22, $24,25]$.

Because leisure activities were related to well-being and physical health was related to well-being in addition 
to leisure activities, leisure activities were tested as a mediator of the relationship between physical health and leisure activities. The results indicated that leisure activities are in part responsible for the relationships between both indicators of physical health and well-being. These findings expand on previous literature by identifying one of the factors linking physical health and well-being. Unlike other factors related to well-being, participating in leisure activities is a lifestyle choice that individuals can modify. For example, individuals may be able to adjust activities when these become more difficult due to health reasons, but whether they do so may be related to their ability to successfully adapt to such challenges. In fact, according to the Lines of Defense model, modifying or even eliminating certain leisure activities in the face of increasing health limitations may be the best strategy to maximize subjective well-being [36].

Our results suggest that leisure activity interventions may be beneficial for adults of all ages. There have been interventions designed to increase leisure activity participation in older adults; however, these have rarely focused on improving well-being [37-40]. If a psychological outcome was a target of an activity intervention, it was mostly to decrease symptoms of depression [41-43]. Therefore, future interventions should seek to enhance wellbeing in adults of all ages in addition to improving symptoms of psychological illnesses; such interventions would benefit the general population and could thus serve as universal, as opposed to targeted, prevention programs. Johnson et al. [44] suggest tailoring activity interventions for older adults based on their readiness to change their activity levels. Given the role that physical health has for activity participation regardless of age in the current study, physical health may be an important, overlooked factor in adults' readiness to change their lifestyles in order to more frequently engage in leisure activities. Therefore, interventions should take into account the physical health of the participants, both objectively and subjectively, in order to potentially increase the likelihood of improving well-being through leisure activities.

\section{Limitations}

Though these findings have important implications for the well-being of adults of all ages, this study has several limitations. The cross-sectional design of the study diminished our ability to infer direction of causality; a longitudinal design would better support the conclusion that leisure activities are in part responsible for the link between physical health and well-being. Furthermore, recent work has demonstrated that cross-sectional media- tion analyses may show a significant indirect effect when there is no true longitudinal effect [45], so the results of the mediation model in the current study should be interpreted with caution. The sample was recruited through responses to advertisements and the participants were paid for their participation, which may have led to a sampling bias. In addition, certain ethnic groups, namely those who were identified as Latino or Asian, were underrepresented in this sample. Future studies should address these sampling issues to increase the generalizability of findings on the importance of leisure activities. Furthermore, it would have been interesting to consider the different types of activities separately, given that health may enable or hinder these activities in different ways (e.g., social vs. physical activities). Yet, we were most interested in examining the overarching relations between activities, health and well-being in the present paper, which is in line with prior studies as well as established approaches in this area, such as activity theory. Future analysis should take differential effects into account.

\section{Conclusion}

The results of this study highlight the importance of leisure activities not only for older adults, but also adults of all ages. Adults should be encouraged to engage in leisure activities throughout their lives to maintain and even improve their well-being. Because physical health may limit the types of leisure activities adults can participate in, more interventions should be developed to ensure that a variety of activities are available regardless of physical ability. Research suggests that specific factors found to be important for well-being, such as social engagement and leisure activities, are precursors for aging successfully [3]. Thus, successful aging should be seen as a lifelong process that is to a certain extent modifiable through participating in leisure activities.

References

1 Depp CA, Jeste DV: Definitions and predictors of successful aging: a comprehensive review of larger quantitative studies. Am J Geriatr Psychiatry 2006;14:6-20.

2 Pressman SD, et al: Association of enjoyable leisure activities with psychological and physical well-being. Psychosom Med 2009;71: 725-732.

3 Pruchno RA, Wilson-Genderson M, Cartwright F: A two-factor model of successful aging. J Gerontol B Psychol Sci Soc Sci 2010; 65B:671-679. 
4 Martin P, et al: Defining successful aging: a tangible or elusive concept? Gerontologist 2015;55:14-25.

5 Katz S, Calasanti T: Critical perspectives on successful aging: does it 'appeal more than it illuminates'? Gerontologist 2015;55:26-33.

6 Lawton MP, et al: Motivation in later life: personal projects and well-being. Psychol Aging 2002;17:539-547.

7 Menec VH, Chipperfield JG: Remaining active in later life. The role of locus of control in seniors' leisure activity participation, health, and life satisfaction. J Aging Health 1997;9: 105-125.

8 Taylor-Piliae RE, et al: Validation of the Stanford Brief Activity Survey: examining psychological factors and physical activity levels in older adults. J Phys Act Health 2010;7:87-94.

-9 Jopp DS, Hertzog C: Assessing adult leisure activities: an extension of a self-report activity questionnaire. Psychol Assess 2010;22:108120.

10 Ashe MC, et al: Older adults, chronic disease and leisure-time physical activity. Gerontology 2009;55:64-72.

11 Janke M, Davey A, Kleiber D: Modeling change in older adults' leisure activities. Leis Sci 2006;28:285-303.

$\checkmark 12$ McAuley E, et al: Trajectory of declines in physical activity in community-dwelling older women: social cognitive influences. J Gerontol B Psychol Sci Soc Sci 2009;64B:543550.

$\checkmark 13$ Moschny A, et al: Barriers to physical activity in older adults in Germany: a cross-sectional study. Int J Behav Nutr Phys Act 2011;8:121.

14 Haley C, Andel R: Correlates of physical activity participation in community-dwelling older adults. J Aging Phys Act 2010;18:375-389.

$\checkmark 15$ Hildebrand M, Brewer M, Wolf T: The impact of mild stroke on participation in physical fitness activities. Stroke Res Treat 2012;2012: 548682.

16 Jefferis BJ, et al: Adherence to physical activity guidelines in older adults, using objectively measured physical activity in a populationbased study. BMC Public Health 2014;14:382.

17 Zimmer Z, Lin HS: Leisure activity and wellbeing among the elderly in Taiwan: testing hypotheses in an Asian setting. J Cross Cult Gerontol 1996;11:167-186.

18 Menec VH: The relation between everyday activities and successful aging: a 6-year longitudinal study. J Gerontol B Psychol Sci Soc Sci 2003;58:S74-S82.
9 Kahana E, et al: Altruism, helping, and volunteering: pathways to well-being in late life. J Aging Health 2013;25:159-187.

20 Van Willigen M: Differential benefits of volunteering across the life course. J Gerontol B Psychol Sci Soc Sci 2000;55:S308-S318.

21 Tsaousis I, et al: Do the core self-evaluations moderate the relationship between subjective well-being and physical and psychological health? Pers Individ Dif 2007;42:1441-1452.

22 Grant N, Wardle J, Steptoe A: The relationship between life satisfaction and health behavior: a cross-cultural analysis of young adults. Int J Behav Med 2009;16:259-268.

23 Peltzer K, Pengpid S: Subjective happiness and health behavior among a sample of university students in India. Soc Behav Pers 2013; 41:1045-1056.

24 Lee A, Browne MO: Subjective well-being, sociodemographic factors, mental and physical health of rural residents. Aust J Rural Health 2008;16:290-296.

25 Barger SD, Donoho CJ, Wayment HA: The relative contributions of race/ethnicity, socioeconomic status, health, and social relationships to life satisfaction in the United States. Quality Life Res 2009;18:179-189.

26 Havighurst RJ, Neugarten BL, Tobin SS: Disengagement and patterns of aging; in Neugarten BL (ed): Middle Age and Aging. Chicago, University of Chicago Press, 1968, pp 161172.

27 Gana K, et al: Does life satisfaction change in old age: results from an 8-year longitudinal study. J Gerontol B Psychol Sci Soc Sci 2013; 68:540-552.

28 Hultsch DF, et al: Memory Change in the Aged. New York, Cambridge University Press, 1998.

29 Pavot WG, et al: Further validation of the Satisfaction with Life Scale: evidence for the cross-method convergence of well-being measures. J Pers Assess 1991;57:149-161.

30 Shaw BA, et al: Age differences and social stratification in the long-term trajectories of leisure-time physical activity. J Gerontol B Psychol Sci Soc Sci 2010;65B:756-766.

31 House JS, Lantz PM, Herd P: Continuity and change in the social stratification of aging and health over the life course: evidence from a nationally representative longitudinal study from 1986 to 2001/2002 (Americans' Changing Lives Study). J Gerontol B Psychol Sci Soc Sci 2005;60B:15-26.

32 Preacher KJ, Hayes AF: SPSS and SAS procedures for estimating indirect effects in simple mediation models. Behav Res Methods Instrum Comput 2004;36:717-731.
33 Jopp DS, et al: Physical, cognitive, social and mental health in near-centenarians and centenarians living in New York City: findings from the Fordham Centenarian Study. BMC Geriatr 2016;16:1.

34 Blomstrand A, et al: Effects of leisure-time physical activity on well-being among women: a 32-year perspective. Scand J Public Health 2009;37:706-712.

35 Kunzmann U, Little TD, Smith J: Is age-related stability of subjective well-being a paradox? Cross-sectional and longitudinal evidence from the Berlin Aging Study. Psychol Aging 2000;15:511-526.

36 Heckhausen J, Wrosch C, Schulz R: A linesof-defense model for managing health threats: a review. Gerontology 2013;59:438-447.

37 Dechamps A, et al: Effects of exercise programs to prevent decline in health-related quality of life in highly deconditioned institutionalized elderly persons: a randomized controlled trial. Arch Intern Med 2010;170:162169.

38 Koizumi D, et al: Efficacy of an accelerometer-guided physical activity intervention in community-dwelling older women. J Phys Act Health 2009;6:467-474.

39 Misic MM, et al: Impact of training modality on strength and physical function in older adults. Gerontology 2009;55:411-416.

40 Tan EJ, et al: The long-term relationship between high-intensity volunteering and physical activity in older African American women. J Gerontol B Psychol Sci Soc Sci 2009; 64B:304-311.

41 Meeks S, et al: BE-ACTIV: a staff-assisted behavioral intervention for depression in nursing homes. Gerontologist 2008;48:105.

42 Motl RW, et al: Depressive symptoms among older adults: long-term reduction after a physical activity intervention. J Behav Med 2005;28:385-394.

43 Tsang HWH, et al: Effect of a qigong exercise programme on elderly with depression. Int J Geriatr Psychiatry 2006;21:890-897.

44 Johnson NL, Hodges JS, Keller MJ: Get moving and keep moving: motivating older adults for participation in leisure time physical activity. Act Adapt Aging 2007;31:57-71.

45 Maxwell SE, Cole DA, Mitchell MA: Bias in cross-sectional analyses of longitudinal mediation: partial and complete mediation under an autoregressive model. Multivariate Behav Res 2011;46:816-841. 